Service social

\title{
Les politiques sociales : des ordres conçus aux ordres vécus
}

\section{Jean-Louis Gendron}

Volume 38, numéro 2-3, 1989

Les politiques sociales

URI : https://id.erudit.org/iderudit/706435ar

DOI : https://doi.org/10.7202/706435ar

Aller au sommaire du numéro

Éditeur(s)

École de service social de l'Université Laval

ISSN

1708-1734 (numérique)

Découvrir la revue

Citer ce document

Gendron, J.-L. (1989). Les politiques sociales : des ordres conçus aux ordres

vécus. Service social, 38(2-3), 155-159. https://doi.org/10.7202/706435ar d'utilisation que vous pouvez consulter en ligne.

https://apropos.erudit.org/fr/usagers/politique-dutilisation/ 


\section{AVANT-PROPOS}

\section{Les politiques sociales : des ordres conçus aux ordres vécus}

Depuis sa fondation, en 1951, jusqu'à aujourd'hui, Service social a toujours laissé un espace de choix à l'analyse des politiques sociales. Certains des articles qui composent le présent numéro le rappellent d'ailleurs avec justesse et pertinence. Même si ce fut à intervalle irrégulier et de façon souvent sporadique, la place laissée à l'analyse des politiques sociales fut importante. Tantôt en éditorial, tantôt dans un commentaire critique, tantôt dans un article à caractère analytique, chaque numéro, ou presque, témoigne du bouillonnement des idées qui entourent l'évolution des politiques sociales au Québec.

Yves Vaillancourt dans son ouvrage L'évolution des politiques sociales au Québec : 1940-1960 dont il est fait mention plus loin, se réfère d'ailleurs abondamment à Service social comme à un témoin de cette époque où les politiques sociales québécoises sont sorties, balbutiantes, de leur berceau. Car dans la société québécoise, la petite et la grande histoire des politiques sociales sont omniprésentes. Elles nous renvoient à nos idéaux collectifs, tout autant qu'à nos vécus quotidiens, à nos espoirs nationaux tout autant qu'à nos réalités personnelles et familiales. En publiant un plein numéro sur les politiques sociales, Service social vient à nouveau témoigner de leur importance.

Mais jusqu'à quel point la façon dont la société québécoise a conçu et conçoit encore ses diverses structures de politiques sociales correspond-elle à la réalité vécue de ces dernières ? Quelle est la distance entre les mythes, les lois et les pratiques ? Voilà les questions auxquelles la plupart des articles présentés ici tenteront de répondre, nous renvoyant sans cesse à la célèbre distinction de Claude LéviStrauss entre les ordres conçus et les ordres vécus.

Ce que Lévi-Strauss appelle des ordres, ce sont des arrangements sociaux particuliers, des structures particulières qui imposent certains 
types de comportements particuliers. Par exemple, une structure hiérarchique dans un système social donné pourrait correspondre à l'un de ces ordres et imposerait alors par le fait même certains types de comportements particuliers, au moment d'une décision. " $\mathrm{Si} \mathrm{A}$, écrira Lévi-Strauss, est supérieur à $B$, et $B$ supérieur à $C, A$ doit être supérieur à $C$, et $C$ ne peut pas être supérieur à $A$ ". (Lévi-Strauss, 1958, p. 346). C'est ce premier type d'arrangements sociaux découlant d'une réalité objective, empiriquement observable, que l'on appellera les ordres vécus.

Mais ce que l'anthropologue a également observé, c'est qu'un deuxième système de règles peut se développer, en plus du premier. Ce sont des ordres qu'il qualifie de "rituels" ou "idéaux". (LéviStrauss, 1958, p. 347). Toutefois, contrairement aux structures d'ordre vécu, ces structures d'ordre conçu ne correspondent directement à aucune réalité objective. Comme d'autres auteurs l'ont par la suite démontré (Lemieux, 1982), ces ordres conçus qui peuvent être soit institutionnels, comme le sont les lois, ou idéaux, comme le sont les représentations idéologiques ou utopiques, nous renvoient aux aspirations, aux valeurs et aux volontés politiques à partir desquelles une société réussit à faire tenir ensemble la structure effective de ses ordres vécus.

Mesurer et interpréter l'écart entre les ordres conçus et les ordres vécus, tel est le but ultime d'une réflexion sur les politiques sociales. Tel est aussi l'apport des auteurs qui offrent ici leur contribution à Service social.

L'objet de leurs observations et de leurs analyses, c'est l'ensemble complet de ce qu'il est convenu d'appeler le réseau des affaires sociales. Ce vaste ensemble enveloppe des organisations particulières, des stratifications sociales et économiques, des structures de pouvoir, etc. qui, chacune à leur façon, offrent des points de repère à l'observateur pour qu'il saisisse l'ordre des réalités sociales qu'elles recouvrent. C'est à cette saisie des réalités structurelles des politiques sociales que nous convie ce numéro de Service social.

D'entrée de jeu, Peter Southam ouvre une perspective historique : comment la notion d'État-providence a-t-elle pris racine au Québec entre le début des années 1930 et le milieu des années 1960 ? En examinant les deux principales thèses sur lesquelles s'appuient les réponses habituelles, soit la thèse du retard et la thèse de la modernisation sans heurt, l'auteur du premier article analyse les paramètres du discours tenu pendant trois décennies, pour montrer comment l'expérience québécoise est à la fois ressemblante mais aussi divergente des autres sociétés occidentales. S'il y a crise de l'État-providence, écrira-t-il, "il apparaît que les diagnostics qui soustendent les constats de crise divergent et se contredisent au point où 
l'observateur se demande si la véritable crise ne se situerait pas au niveau des définitions et des perceptions plus que dans les structures mêmes du régime ». Comme si, au-delà des ordres vécus, la véritable négociation entre les acteurs prenait racine dans des idéaux, des arrangements sociaux virtuels ou des ordres conçus, de nature idéale plus qu'institutionnelle.

L'article de Vincent Lemieux, par ailleurs, attire la réflexion sur la question du pouvoir dans la réalisation des politiques sociales, donc davantage sur les ordres vécus. Comment analyser les politiques sociales de façon à être capable d'agir sur elles, au besoin ? Le politologue s'adresse ici à l'intervenant social et ouvre le dialogue avec lui. II propose " que les phénomènes de pouvoir doivent être au cœur d'une telle démarche analytique, parce que la réalisation ou la non-réalisation des politiques publiques dépend de l'exercice du pouvoir ». Trois types d'acteurs participent à l'exercice du pouvoir : "les responsables", qui dirigent les systèmes, "les agents » parmi lesquels se retrouvent majoritairement les travailleurs sociaux, et " les intéressés " qui constituent la clientèle des organisations où œuvrent ces derniers. C'est grâce aux alliances qu'ils peuvent faire que ces acteurs influencent l'évolution des politiques sociales. En analysant plus particulièrement le cas des CLSC, Vincent Lemieux montre comment ces alliances constituent l'élément fondamental pour exercer du pouvoir dans la réalisation des politiques sociales et comment certains acteurs savent, mieux que d'autres, concocter ces alliances et exercer leur pouvoir.

Suit l'article de Réjean Landry et Paule Duchesneau : "À qui profitent les lois de la mission sociale ? " Partant des lois sociales adoptées par l'Assemblée nationale du Québec entre 1960 et 1985 , l'article est une analyse comparative de l'action gouvernementale en cette matière. Recherche empirique à caractère quantitatif, la démarche des auteurs s'inspire de la théorie des biens publics, pour parler ensuite de la mission sociale de l'État, avant de présenter et analyser les résultats d'une étude conduite sur le terrain. Ce sont cette fois les ordres conçus de nature institutionnelle qui retiennent l'attention.

Ce sont encore les ordres conçus de nature institutionnelle qui inspirent Jean Turgeon dans sa réflexion sur la décentralisation vers les Conseils Régionaux de la Santé et des Services Sociaux (CRSSS). Partant d'une grille à cinq niveaux où il distingue différents types de substrats (ressources monétaires, humaines, matérielles et informationnelles) qui circulent entre l'État et les CRSSS depuis le début de leur existence, l'auteur tente de déterminer l'importance relative de chacun de ces substrats par rapport à un objectif de décentralisation. En conclusion, l'article obligera le lecteur à constater qu'en matière 
de décentralisation des politiques sociales, il y a loin de la coupe aux lèvres...

Puis, vient I'article de Gérald Doré et Lorraine Gaudreau mettant en rapport la politique sociale et l'engagement politique partisan des travailleurs sociaux. L'article rappelle que les politiques sociales s'élaborent dans un processus complexe comportant plusieurs phases ou moments dont celui de la prestation du service où se retrouvent la grande majorité des travailleurs sociaux. Mais d'autres phases existent aussi qui appartiennent au champ de l'action politique partisane. Les travailleurs sociaux y sont-ils présents ? Participent-ils aux jeux des idées, aux stratégies politiques et aux rapports de force d'où émanent les politiques sociales, avant la prestation des services ? Éminemment présents dans la structure des ordres vécus, les travailleurs sociaux - ceux du Québec, en tout cas - ne semblent pas avoir trouvé la voie d'accès pour influencer la formulation des ordres conçus.

Et c'est à ceux-là que le commentaire de Jean-Pierre Deslauriers fait appel dans le premier article de la section "Commentaires et documents ". "Honneur aux rebelles!" s'écrie-t-il, dans son style à l'emporte-pièce ! Honneur à celles des travailleuses sociales - et le féminin n'est pas utilisé par hasard ! - qui cherchent à concevoir un ordre des choses différent dans l'élaboration et la diffusion des politiques sociales. Ce sont, cette fois, les ordres conçus de nature idéale auxquels l'auteur se réfère.

Suivent, finalement, trois articles hors thème qui, sans s'adresser directement à l'étude des politiques sociales, nous y renvoient tout de même de façon indirecte. L'article de Jocelyne Barnabé et Gérald Doré nous propose une méthode pour repérer scientifiquement les zones de pauvreté sur un territoire de CLSC. Celui de Marcelle Laforest et de ses collaborateurs nous présente la réaction du Regroupement des unités de formation universitaire en travail social du Québec (RUFUTS) au rapport de la Commission Rochon. Et, arrivant en dernier malgré toute sa nouveauté, l'article rédigé sous la direction de Jacques Ajenstat nous interroge sur l'utilisation des systèmes experts d'aide à l'intervenant social.

Ce numéro de la revue Service social se situe donc au carrefour de l'analyse politique et de l'intervention sociale, au carrefour de la réflexion et de l'action. Mettant à contribution l'apport de plusieurs disciplines des sciences humaines, il s'adresse à tous ceux qu'inquiète et intéresse l'évolution actuelle des politiques sociales, qu'il s'agisse de les concevoir ou de les vivre. 


\section{Références}

LÉvi-Strauss, Claude, Anthropologie structurale, Plon, Paris, 1958.

LEMIEUX, Vincent, "L'analyse structurale des lois ", Revue canadienne de science politique, XV, 1, mars $1982: 67-84$.

VaILlancourt, Yves, L'évolution des politiques sociales au Québec : 1940-1960, P.U.M., Montréal, 1988. 